\title{
EFFECT OF KINETIC RETURN ANKLE FOOT ORTHOSIS IN PATIENT WITH INCOMPLETE SPINAL CORD INJURY: CHANGES OF THE GAIT PATTERN. A CASE REPORT
}

\author{
Margot Bergmann $^{1}$, Mari Alvela ${ }^{1}$, Priit Eelmäe ${ }^{1,2}$, Doris Vahtrik ${ }^{3}$, Helena Gapeyeva ${ }^{3}$ \\ Haapsalu Neurological Rehabilitation Centrel, Haapsalu, Estonia \\ Centre of Excellence in Health Promotion and Rehabilitation ${ }^{2}$, Haapsalu, Estonia \\ Institute of Sport Sciences and Physiotherapy, University of Tartu³, Tartu, Estonia
}

\begin{abstract}
Background. The variety of orthotics available induces a purpose for estimation of their influence of functional mobility for individual needs in people with incomplete spinal cord injuries (ISCI). The aim of the study was to investigate the effect of the use of kinetic return ankle foot orthosis (KRAFO) on gait pattern in case of ISCI.

Methods. Ankle and knee joint kinematic and kinetic characteristics during gait with and without KRAFO were studied in a 34-year-old man with ISCI (fracture v.C5) using 3D motion analysis system (Vicon Motion Systems Ltd., UK) including two dynamographic platforms (AMTI, USA). Ankle and knee joint angles at initial contact and mid-stance, ankle dorsiflexion and foot progression angle in swing phase and ankle joint push-off values in stance phase were analysed.

Results. An excessive dorsiflexion in right ankle joint at initial contact, in mid-stance and in swing-phase occurred when walking without the orthosis, which decreased $(105,57$ and $73 \%$, respectively, $p<.01)$ with the use of KRAFO. Orthoses use evoked the decrease $(77 \%, p<.01)$ in peak foot progression angle. Ankle joint peak pushoff power was low without the use of KRAFO and decreased even more $(28 \%, p<.05)$ with the use of orthosis. Decreases of knee joint flexion angle at initial contact and in mid-stance (29 and 23\%, respectively) with the use of KRAFO were not significant as compared to gait without orthosis.

Conclusions. Walking with KRAFO improved ankle and knee joint stability, providing a decrease in ankle kinematic characteristics but ankle joint push-off power did not change. Further studies are needed to compare the effect of KRAFO in comparison with other orthoses on gait pattern in case of ISCI in accordance with the patientcentric approach for rehabilitation process management.
\end{abstract} of gait.

Keywords: spinal cord injury, clinical gait analysis, ankle foot orthosis, kinematic and kinetic characteristics

\section{INTRODUCTION}

$O$ pinal cord injury (SCI) is a traumatic event that affects conduction of both sensory and motor signals across the site of lesion. Autonomic nervous system could be affected as well; therefore patients' physical, psychological and social well-being is interrupted (Kirshblum et al., 2011; Singh, Tetreault, Kalsi-Ryan, Nouri $\&$ Fehlings, 2014). One of the complications after the injury is skeletal muscle atrophy. Smaller cross-sectional area is related to the loss of central activation and insufficient loading. The chronic stages of injury also include connective tissue infiltration in skeletal muscles (Gorgey \& Dudley, 2007).

The ability to restore gait function is the "ultimate goal" for incomplete spinal cord injured patients (ISCI) and the ability to just stand is not enough. However, if patients are able to walk independently, they usually want to improve the quality of their gait (van der Salm et al., 2005). 
A large amount of impairments that affect the gait pattern of ISCI patients are related to foot clearance problems in swing phase, i.e. excessive plantarflexion, limited knee or hip flexion, eversion of ankle joint. Due to the clearance problems, initial contact is also impaired. In the case of typical gait pattern, ankle joint is dorsiflexed to a neutral position in terminal swing, so that heel strike can occur at initial contact. If plantarflexion in swing phase is present, compensatory movements (i.e. unstable pelvic movement, compensatory functions of contralateral leg) are performed to rise foot from the floor (van der Salm et al., 2005). Patients with central neurological disorders have also reduced ability to push off from the floor with ankle joint. Low push-off values are caused by weakness of plantarflexors. An excessive work by hip joint could be used to compensate this reduced ability to push off (Bregman, Harlaar, Meskers \& de Groot, 2012). During initial and mid-swing phases, changes are caused by stiffness of plantarflexors and also by weakness of ankle dorsiflexors. During terminal swing, in addition to ankle joint position, the position of whole lower limb could be affected by a decrease in angular velocity that is generated by hip flexors (Barbeau, Ladouceur, Mirbagheri \& Kearney, 2002).

A conventional approach to correct drop-foot is custom-made ankle-foot orthotics (AFO), that keeps ankle joint in neutral position and does not allow the foot to drop in swing phase of gait (Kottink et al., 2004; Zou et al., 2014). One of the most used AFOs is posterior leaf spring (PLS) AFO, which is made of thermoplastics. PLS AFOs have very low energy storage and energy return capabilities; therefore they do not help patients during propulsion and do not generate enough energy to push off from the ground (Zou et al., 2014). Use of PLS AFO may contribute to disuse atrophy of calf muscles (Meier, Ruthsatz \& Cipriani, 2014). AFO immobilizes inversion, eversion, abduction, adduction and plantarflexion, allowing only dorsiflexion of ankle joint, so AFOs with support around foot and/or ankle can improve medio-lateral stability of ankle during stance phase (Meier et al., 2014; Slijper, Danielsson \& Willen, 2014). As the use of AFO prevents drop-foot, significant reduction in muscle activity of tibialis anterior is also noticed while wearing the orthosis; therefore prolonged usage could lead to atrophy (Meier et al., 2014).

Nowadays modern orthotics are available that provide more dynamic design - a kinetic return AFO
(KRAFO) (ToeOFF®, Allard, USA) (Figure 1). Using KRAFO allows normal biomechanical function in talocalcaneal and talotarsal joints compared to PLS AFO, which causes additional calf muscle activity during gait (Meier et al., 2014). Prefabricated KRAFOs are used to stabilize ankle joint in anterio-posterior direction (Slijper et al., 2014). Also KRAFOs return energy during third rocker of gait to assist with propulsion, which also causes more muscle activity in plantarflexors (Meier et al., 2014).

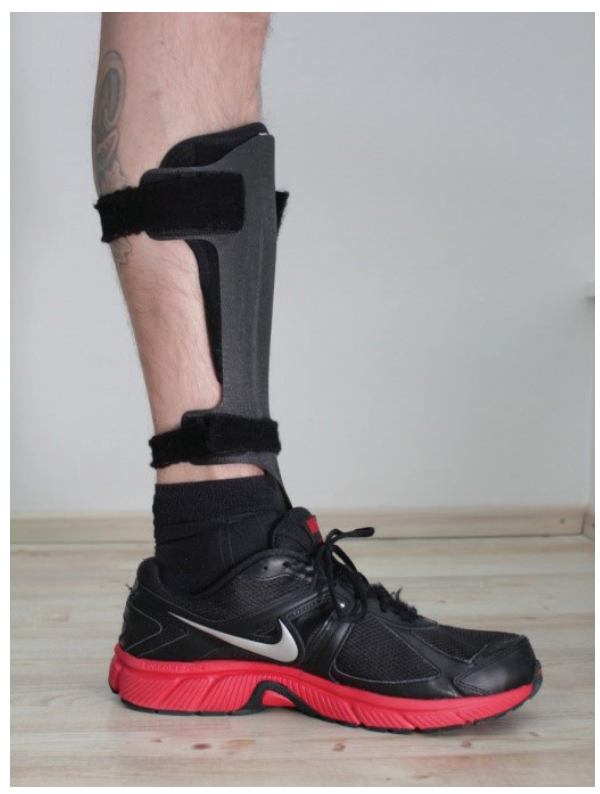

Figure 1. Kinetic return ankle foot orthosis

KRAFOs are also known as carbon-composite AFOs. Among other things, KRAFOs can be used to improve ankle joint power and plantarflexor moment (Bregman et al., 2012; Zou et al., 2014). KRAFO has properties that enable storage of energy at the begging of stance phase and a return of this energy at the end of stance phase. The return of energy in late stance phase should increase push-off values and therefore reduce the need for different compensation strategies (Bregman et al., 2012)

Instrumented clinical gait analysis (CGA) that is conducted in laboratory is more precise than observational gait analysis as objective information could be collected, such as electromyography, kinetic and kinematic data. Joint movement could be observed in all three planes and forces that act on the body are recorded by dynamographic platforms. All the collected data is compared at the exactly same percentage of gait cycle (Chambers \& Sutherland, 2002). 
This case study was conducted to evaluate whether using KRAFO improves gait pattern of ISCI patient. As carbon-composite orthosis are relatively new, only few studies have been conducted. KRAFO is probably a proper orthosis also for patients with ISCI, as it is for other central neurological disorders that have been researched already (Bregman et al., 2012). As the main deviations of gait in ISCI patients are impaired initial contact, drop-foot in swing and low pushoff values in the end stance phase, KRAFO could be a proper solution for this patient group as it is expected to solve all above mentioned deviations.

The aim of the current study was to evaluate ankle and knee joint angles at initial contact and mid-stance, ankle dorsiflexion and foot progression angle in swing phase and ankle joint push-off power in the end of stance phase during gait with and without kinetic return ankle foot orthosis in patients with ISCI. We hypothesized that while using KRAFO (1) it would be possible to keep ankle joint in neutral position during initial contact and mid-stance and therefore to reduce semiflexion in knee joint; (2) an excessive ankle joint dorsiflexion and foot progression angle in swing phase would decrease; (3) ankle joint push-off values would increase.

\section{METHODS}

Subject. A man aged 34 years with traumatic ISCI (fracture of $5^{\text {th }}$ cervical vertebrae), American Spinal Injury Association Impairment Scale (AIS) grade D (motor incomplete) volunteered to participate in the present study. Patient's body mass was $90 \mathrm{~kg}$, height $200 \mathrm{~cm}$ and body mass index $22.5 \mathrm{~kg} / \mathrm{m}^{2}$. Time since injury was 4.5 years at the moment of current analysis. The patient was able to walk independently and did not need any walking aids. KRAFO was used to correct his gait pattern for about a week. The patient wore KRAFO for approximately 12 hours per day. Prior to injury, the patient was an amateur athlete (extreme sports), with training load of 6-8 hours per week. The patient read and signed a written informed consent form in regard to the Declaration of Helsinki principles. The study had an approval from the ethics committee.

Measurement procedures. Prior to gait analysis, a thorough physical therapy assessment was carried out by two physiotherapists (PT) - all of the measurements were carried out by the same therapist and assisted by other. Muscle strength of lower extremities was assessed using Modified Oxford Scale (min grade 0/5 - no contraction; max grade 5/5 - movement against gravity with full resistance). Length of hamstring muscles was assessed by popliteal angle and length of plantarflexors by Silverskjöld test. Modified Tardieu scale was used to measure muscle tone of knee extensors and flexors and of plantarflexors (min T0 - no resistance throughout passive movement; max T4 - unfatigable clonus ( $>10 \mathrm{~s}$ ) occurring at a precise angle; R1/R2 - spasticity angle; R1 - angle of catch seen at fast velocity; R2 - full range of motion achieved when muscle is at rest and tested at slow velocity) ( $\mathrm{Li}, \mathrm{Wu} \& \mathrm{Li}$, 2014). Goniometer was used to measure joint range of motion (ROM). Leg length was measured with non-elastic tape from anterior superior iliac spine to medial malleolus. Body balance was assessed by three tests: Tinetti Balance Assessment Tool ( $\leq 18$ high; $19-23$ moderate; $\geq 24$ low risk of falls) (Vaught, 2001), Romberg's test and standing on one leg. Selective motor control (SMC) of tibialis anterior muscle was assessed with modified Trost scale (min grade 0 - only patterned movement observed, total synergy; max grade 2 - completely isolated movement observed, no synergy) (Zwaan, Becher \& Harlaar, 2012). After PT assessment, instrumented CGA was conducted firstly with regular shoes and afterwards using KRAFO on right foot. All the measurements and data capturing were conducted within one day.

Kinematics and kinetics of gait. Threedimensional gait analysis system with eight infrared cameras (MX-T20 cameras, $100 \mathrm{~Hz}$; Vicon Motion Systems Ltd., UK) was used to capture kinematic data and two dynamographic platforms (AMTI, USA) were used to capture kinetic data. In addition two video cameras (Basler, USA) were used to capture video data from sagittal and coronal planes. Data was captured with Nexus 1.4.1. software and presented for interpretation with Polygon 3.1. software (Vicon Motion Systems Ltd., UK). Reflective markers were placed according to Davis model (Baker, 2006). The patient walked over-ground on an eight meter walkway. Fifteen trials were performed for each condition - gait with and without KRAFO and mean data was used for analysis. Duration of resting pause between trials was 3 to 5 minutes, when the patient sat on a spinesupporting chair.

Statistical analysis. MS Excel software was used to analyse the gait characteristics. Data are arithmetic means and standard deviations $(S D)$. 
Outcome parameters during walking with regular shoes and with KRAFO were compared using Student's paired samples $t$-test. Change (in percent) for outcome parameters as compared to initial level was calculated applying the formula $(b-a) / a$, where $a$ presents data for walking without orthosis and $b$ - data for walking with use of the KRAFO. The lowest level of significance was set to $p<.05$

\section{RESULTS}

Pre-testing patient evaluation. Data of the patient's pre-testing investigation is presented in Table 1.

The subject had $2 \mathrm{~cm}$ leg length discrepancy (right $>$ left). The obtained data demonstrated an increase in Popliteal angle and therefore a decrease in length of hamstring muscles bilaterally. Length of gastrocnemius muscles was also decreased bilaterally, which was measured using Silverskjöld test. Spasticity was greater distally than proximally; clonus occurred in soleus and gastrocnemius muscles. Muscle strength was in moderate level ranging from grade four to five. Right tibialis anterior muscle had impaired selective motor control. Body balance impairment was found while eyes were closed or standing on right leg, but overall falling risk was estimated as low.

Ankle joint characteristics during gait. Characteristics of ankle and knee joint during walking with and without the use of KRAFO are presented in Table 2 and the example of change of ankle joint kinematics in sagittal and transversal planes is demonstrated in Figure 2.

At initial contact, an excessive dorsiflexion of right ankle joint was noted while walking with regular shoes, which decreased with using KRAFO. Dorsiflexion angle was reduced by $10.4^{\circ}$, which is $105 \%$ less $(p<.01)$ than angle with regular shoes while walking with KRAFO.

When walking without KRAFO, an apparent dorsiflexion in mid-stance in right ankle joint was found. Use of KRAFO reduced dorsiflexion angle by $10.4^{\circ}$ in ankle joint in the middle of stance phase, which is $57 \%$ less $(p<.01)$ than angle without orthosis.

The patient demonstrated an excessive dorsiflexion in swing phase in right ankle joint; peak dorsiflexion in swing phase exceeded reference values while walking without KRAFO.

\begin{tabular}{|c|c|c|}
\hline Measure & Sin & Dex \\
\hline Leg length discrepancy (cm) & \multicolumn{2}{|c|}{$2(\operatorname{dex}>\sin )$} \\
\hline Popliteal angle $\left({ }^{\circ}\right)$ & 40 & 50 \\
\hline Silverskjöld test $\left(\right.$ knee $\left.90^{\circ} \mathbf{0}^{\circ}\right)$ & $(25 / 0)$ & $(20 /-5)$ \\
\hline \multicolumn{3}{|l|}{ Spasticity $\left(\mathrm{R} 1^{\circ} / \mathbf{R 2}^{\circ}\right)$} \\
\hline Rectus femoris muscle & T0 & $\mathrm{T} 2(30 / 150)$ \\
\hline Hamstrings & T2 $(90 / 100)$ & $\mathrm{T} 2(55 / 90)$ \\
\hline Soleus muscle & $\mathrm{T} 3$ & $\mathrm{~T} 4$ \\
\hline Gastrocnemius muscle & $\mathrm{T} 3$ & $\mathrm{~T} 3$ \\
\hline \multicolumn{3}{|l|}{ Muscle strength (points) } \\
\hline Knee Extensors & 5 & 5 \\
\hline Hamstrings & 5 & 5 \\
\hline Dorsiflexors & 5 & $4+$ \\
\hline Plantarflexors & 5 & 4 \\
\hline Selective motor control of tibialis anterior muscle (points) & 2 & 1 \\
\hline Ankle instability & + & + \\
\hline \multicolumn{3}{|l|}{ Balance } \\
\hline Tinetti test (points) & \multicolumn{2}{|c|}{ Score 25} \\
\hline Romberg test & \multicolumn{2}{|c|}{ Eyes closed unsecure } \\
\hline Standing on one leg (s) & OK & 5 (compensating) \\
\hline
\end{tabular}


Table 2. Gait characteristics of ankle and knee joints with and without the orthosis in incomplete spinal cord injured patient

\begin{tabular}{|c|c|c|c|c|c|}
\hline Gait characteristic & $\begin{array}{c}\text { Without KRAFO } \\
(\text { mean } \pm S D)\end{array}$ & $\begin{array}{c}\text { With KRAFO } \\
(\text { mean } \pm S D)\end{array}$ & $\begin{array}{l}\text { Percentages } \\
(\%)\end{array}$ & Change & $p$-value \\
\hline \multicolumn{6}{|l|}{ Ankle joint angles $\left({ }^{\circ}\right)$} \\
\hline Dorsiflexion at initial contact & $9.9 \pm 2.7$ & $-0.5 \pm 1.1$ & 105 & $\downarrow$ & .003 \\
\hline Dorsiflexion at mid-stance & $18.3 \pm 2.3$ & $7.9 \pm 1.6$ & 57 & $\downarrow$ & .003 \\
\hline Dorsiflexion max in swing & $19.8 \pm 0.3$ & $5.4 \pm 0.5$ & 73 & $\downarrow$ & .001 \\
\hline Foot progression in swing & $44.8 \pm 3.7$ & $10.3 \pm 2.0$ & 77 & $\downarrow$ & .0001 \\
\hline \multicolumn{6}{|l|}{ Knee flexion angles $\left({ }^{\circ}\right)$} \\
\hline Initial contact & $18.2 \pm 1.9$ & $12.9 \pm 5.4$ & 29 & $\downarrow$ & .19 \\
\hline Mid-stance & $15.7 \pm 3.7$ & $12.0 \pm 0.6$ & 23 & $\downarrow$ & .16 \\
\hline \multicolumn{6}{|l|}{ Ankle power (W/kg) } \\
\hline Max power generation & $1.5 \pm 0.2$ & $1.1 \pm 0.1$ & 28 & $\downarrow$ & .03 \\
\hline
\end{tabular}

Note. KRAFO - Kinetic return ankle foot orthosis. Change (\%) for outcome parameters as compared to initial level was calculated by formula $(b-a) / a$, where $a$ presents data for walking without orthosis and $b$ - data for walking with use of the KRAFO. The arrow shows the decrease of gait characteristics with orthosis as compared to without orthosis demonstrating the improvement, except for ankle push-off values.

Figure 2. Ankle joint kinematic characteristics in patient with ISCI: In sagittal plane for dorsal/plantar flexion angle during walking without (A) and with kinetic return ankle foot orthosis (KRAFO) (B); in transversal plane for foot progression angle during walking without (C) and with KRAFO (D)

Note. KRAFO for the right leg was used.

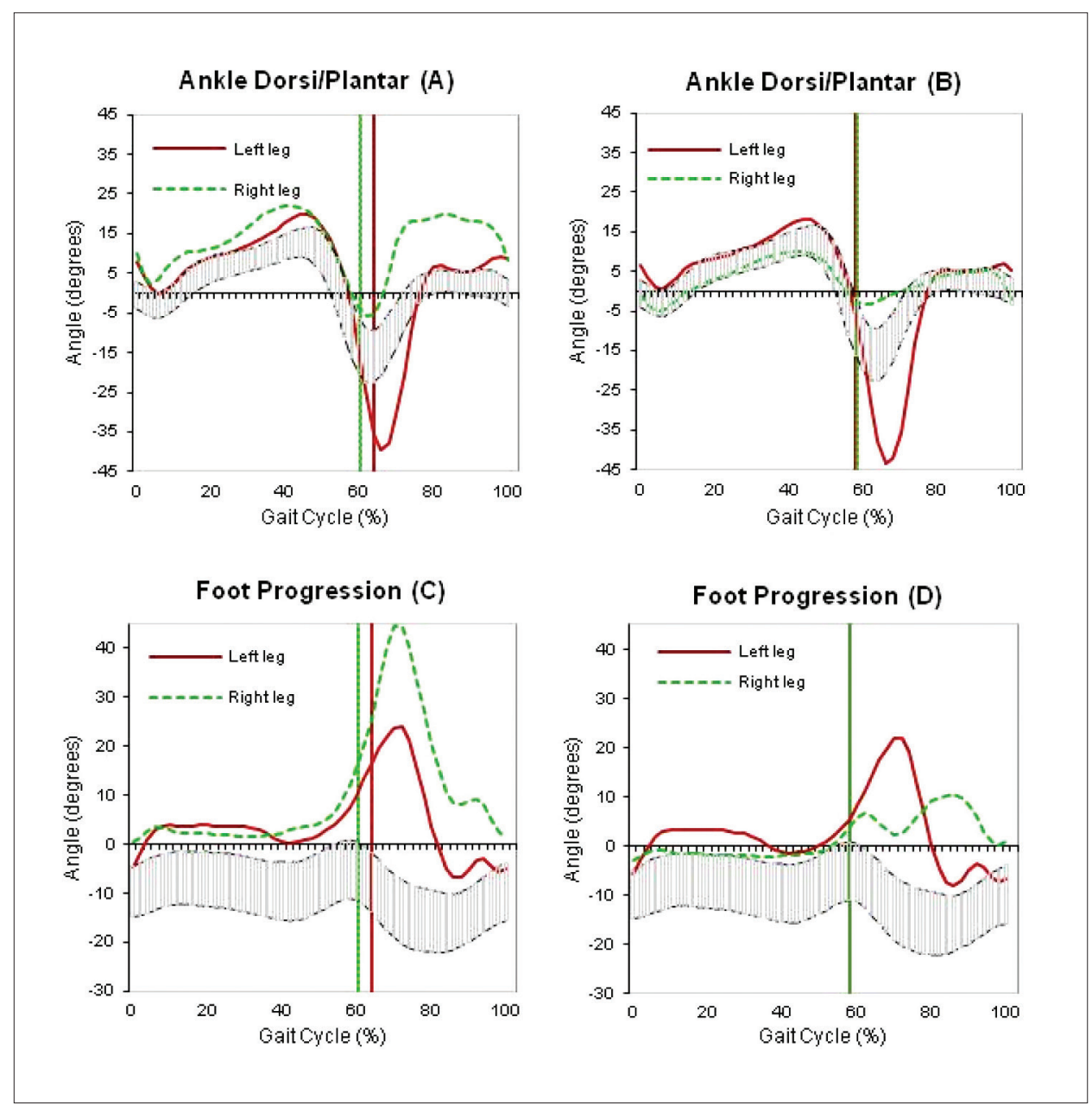

Corresponding dorsiflexion value decreased $14.4^{\circ}$ while walking with orthosis, which is $73 \%$ less $(p<.01)$ than angle without KRAFO.
Instability of ankle joint caused extensive movement towards internal rotation on foot progression characteristics in the beginning of 
swing phase while walking with regular shoes. Using KRAFO improved ankle stability and foot progression angle approached normal values. Peak internal rotation reduced $34.5^{\circ}$ while walking with KRAFO which is $77 \%$ less $(p<.01)$ than peak angle with regular shoes.

The example of change of ankle power characteristics is presented in Figure 3. Push-off values in ankle joint were low while walking with regular shoes and decreased even more $(0.4 \mathrm{~W} / \mathrm{kg})$ while walking with orthosis. Peak push-off value decreased by $28 \%(p<.05)$ with the use of KRAFO as compared to walking with regular shoes.

Knee joint characteristics during gait. The example of change of knee joint kinematics in sagittal plane is demonstrated in Figure 4. Initially contact semiflexion occurred in knee joint, which was reduced to some extent $\left(5.3^{\circ}\right)$ while walking with KRAFO, which is $29 \%$ less $(p>.05)$ than walking with regular shoes.
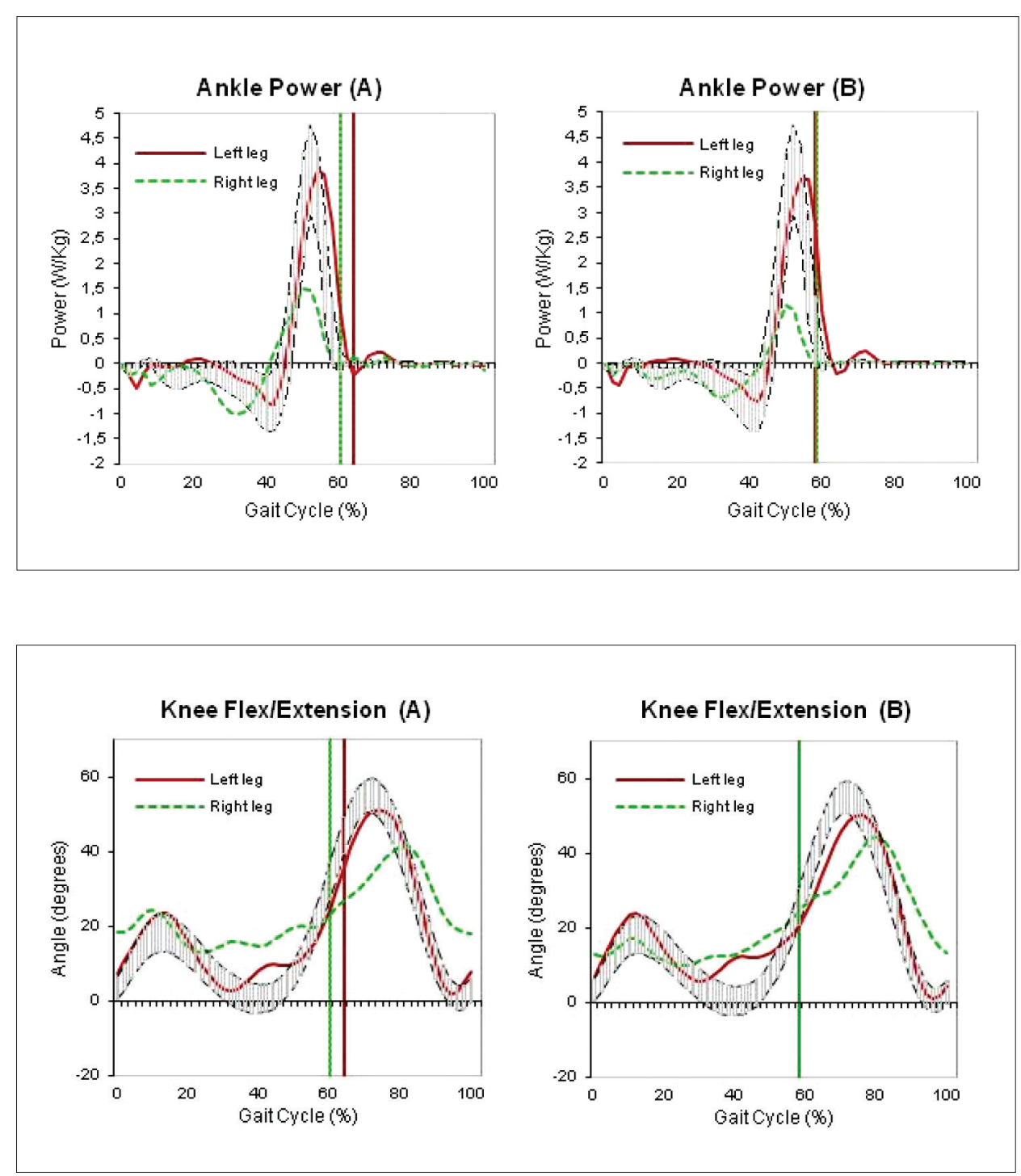

Figure 3. Ankle joint power characteristics in patient with ISCI: Push-off values during walking without (A) and with kinetic return ankle foot orthosis (KRAFO) (B)

Note. KRAFO for the right leg was used.

Figure 4. Knee joint kinematic characteristics in sagittal plane in patient with ISCI: Flexion/extension angle during walking without $(\mathrm{A})$ and with kinetic return ankle foot orthosis (KRAFO) (B)

Note. KRAFO for the right leg was used.
Knee joint was in semiflexion also in midstance while walking without orthosis. Knee flexion values in mid-stance while walking with KRAFO decreased by $3.7^{\circ}$ which is $23 \%$ less ( $p>$ $.05)$ as compared to gait without orthosis, but it did not completely reach reference values. The changes of knee joint range of movement characteristics were not statistically significant.

\section{DISCUSSION}

The current study was conducted to evaluate the acute effects of carbon-composite KRAFO in ISCI patient. The main focus was on ankle joint range of motion in sagittal and transverse planes; knee joint ROM in sagittal plain and also on ankle power changes. The main findings of the study 
were that using KRAFO in a patient with ISCI (1) reduced ankle joint dorsiflexion and knee joint flexion at initial contact and mid-stance; (2) reduced ankle joint dorsiflexion and foot progression angle in swing phase; (3) push-off values of ankle joint did not change significantly.

An excessive dorsiflexion in ankle joint at initial contact was caused by semiflexion in knee joint and also because of the patient's initiative to improve clearance in swing. Semiflexion in knee joint causes an apparent dorsiflexion because tibia is moved anteriorly in relation to foot, causing bigger dorsiflexion ROM. The use of KRAFO helped to reduce dorsiflexion and knee joint flexion at initial contact.

Dorsiflexion in stance phase was also caused by excessive knee flexion. During typical gait pattern, almost full extension should occur in knee joint in mid-stance, but in this case semiflexion occurs instead in both walking types - with and without orthosis. Plantarflexion-knee extension couple (PF$\mathrm{KE}$ ) plays an important role in controlling knee joint. In case of full knee extension, knee extensors become relatively insufficient and control only the first phase of knee extension. The second phase, where maximal knee extension is achieved, is controlled by plantarflexors. If the second phase is missing, it leads to a mild crouch gait, even though quadriceps muscle strength may be normal (Brunner \& Rutz, 2013). In this case the second phase is impaired and PF-KE was insufficient; therefore ground reaction force (GRF) aligned behind the knee joint centre and mild crouch occurred. Plantarflexor muscle activation was insufficient and knee extensor muscle activation was excessive. At loading response and mid-stance, external knee flexion moment is increased, which causes knee extensors to work excessively to avoid collapse. The use of KRAFO in case of patient with ISCI improved PF-KE; GRF aligned more towards the knee joint centre, therefore knee semiflexion in stance phase was reduced causing less dorsiflexion. Also muscle work in stance phase was improved due to better biomechanical alignment.

At terminal stance and pre-swing ankle joint moves towards plantarflexion and eccentric contraction in plantarflexors occur. As the length of right plantarflexor muscles is decreased, not enough energy is stored to the muscles; therefore energy that is generated at push-off is also decreased (Bregman et al., 2012). While walking with KRAFO, plantarflexion ROM is even more limited due to the design of orthosis and plantarflexors do not elongate enough, therefore push-off values of right ankle joint are decreased while walking with orthosis. In this case the patient has moderate strength during plantarflexion and muscles are able to generate some energy, therefore KRAFO may even interrupt muscles to work independently and increase in push-off values is not noticeable. Meier et al. (2014) found that the use of KRAFO improved calf muscle circumference compared to plastic AFO in patients with neurological disorders. For example, plastic AFO immobilises calcaneus in a subtalar neutral position and therefore diminishes functional capacity of the calf muscle group. In our study, the patient had moderate plantarflexion strength and use of KRAFO immobilizes muscles and probably calf muscle group atrophy should be seen if AFO is used for a long time in contrast to Meier et al. (2014) study. Results of our study are in line with study conducted by Bregman et al. (2012) where using spring-like AFO increase in maximum ankle power generation was not found. In their study, data of gait analysis using AFO in patients with neurological deficit who had some ankle joint function with not complete drop-foot was studied. They also state that this type or orthosis may contribute to reduction of calf muscle activity.

Drop-foot that is caused by weakness and/or impairment of selective motor control of muscles is a very common problem in ISCI patients (van der Salm et al., 2005). To avoid tripping and falling, dorsiflexors are activated as much as possible to rise the foot from the ground. In our study an overactivity of dorsiflexors was a cause for excessive dorsiflexion in swing phase - muscles pulled the foot of the ground too much as they had moderate strength and SMC grade 1, so the patient was able to dorsiflex ankle joint and did that excessively to ensure that he did not fall. The use of orthosis does not allow excessive dorsiflexion due to its mechanical properties. Therefore dorsiflexion is reduced in the swing phase and muscles do not have to work excessively to rise the foot from the ground. Ankle joint is kept in a neutral position. Meier et al. (2014) stated that wearing orthosis could cause tibialis anterior muscle atrophy which could be a problem in this case also. The patient is used to activate dorsiflexor muscles excessively to rise the foot, but if he wears orthosis there is no need to activate them anymore. Therefore longer wearing period could cause muscle disuse atrophy.

In transverse plane, foot progression angle in stance phase is turned towards internal rotation to some extent. During toe-off and at the begging 
of swing phase, rapid movement towards internal rotation occurs that reduces in mid-swing. The main causes for the rapid movement are ankle joint instability and modest weakness of dorsiflexor muscles. While walking with KRAFO, foot progression angle moves more towards reference values and rapid movement right after the toeoff and in swing phase is also reduced. Meier et al. (2014) claim that the use of KRAFO allows to improve biomechanical function in talocalcaneal and talotarsal joints, but does not allow an excessive movement; therefore it stabilizes the ankle joint.

The patient has leg length discrepancy (right leg, which is more affected, is $2 \mathrm{~cm}$ longer than left leg), which could also be a reason for deviated gait characteristics. The patient may unconsciously try to compensate the leg length discrepancy by increasing flexion in right leg. This could also be the reason for semiflexion in knee joint and therefore excessive dorsiflexion in ankle joint. As functional leg length was not measured (from navel to medial malleolus), no strong conclusions or recommendations could be made.

Practical impact. The study of acute effects of using a KRAFO demonstrated improved ankle and knee joint flexion in stance phase and improved foot progression and dorsiflexion in swing phase. Using a kinetic return AFO has positive effect on ISCI patient's gait pattern on both stance and swing phases concerning ankle joint kinematics. The study with more subjects could be performed for the evaluation of the effect of KRAFO on kinetic characteristics of ankle joint during gait.

Strengths and limitations of the study. The present study strength feature is the fact that KRAFOs are relatively new and have not been tested on ISCI patients. Our study provides detailed information about the acute effect of KRAFO on ankle and knee joint characteristics. Our pilot study results demonstrate that the use of KRAFO does improve gait characteristics of ISCI patient and provide a good insight for future research with greater patient population.

Limitation of the study is that despite that case gives personal information we cannot generalize the results of the present case study to a wider population. Electromyography should also be conducted to declare that use of KRAFO reduces the activity of calf muscle group.

As the patient did not receive any gait training with orthosis and he had worn it for a short period of time, gait pattern could be affected. Only acute effects of the KRAFO were evaluated. The influence of different orthosis on gait characteristics should have been compared for ISCI patient.

\section{CONCLUSIONS}

It can be concluded that in case of a patient with ISCI, immediate effect of the use of KRAFO demonstrated improvement in ankle joint dorsiflexion and knee joint flexion at initial contact and in mid-stance. The use of KRAFO reduced ankle joint dorsiflexion and foot progression angle at swing phase. However, ankle power at pushoff did not change significantly due to moderate plantarflexor strength and limited function of ankle joint. Further studies are needed to compare the effect of KRAFO in comparison with other orthosis (for example, carbon-composite AFO) on gait pattern in case of ISCI in accordance with the patient-centric approach for management of the rehabilitation process.

\section{REFERENCES}

Baker, R. (2006). Gait analysis methods in rehabilitation. Journal of Neuroengineering and Rehabilitation, 2(3), 4. doi:10.1186/1743-0003-3-4.

Barbeau, H., Ladouceur, M., Mirbagheri, M. M., \& Kearney, R. E. (2002). The effect of locomotor training combined with functional electrical stimulation in chronic spinal cord injured subjects: Walking and reflex studies. Brain Research Reviews, 40, 274-291.

Bregman, D. J. J., Harlaar, J., Meskers, C. G. M., \& de Groot, V. (2012). Spring-like Ankle Foot Orthoses reduce the energy cost of walking by taking over ankle work. Gait \& Posture, 35, 148-153.
Brunner, R., \& Rutz, E. (2012). Biomechanics and muscle function during gait. Journal of Children's Orthopaedics, 7, 367-371. doi: 10.1007/s11832-013-0508-5

Chambers, H. G., \& Sutherland, D. H. (2002). A Practical guide to gait analysis. Journal of the American Academy of Orthopaedic Surgeons, 10(3), 222-231.

Gorgey, A. S., \& Dudley, G. A. (2007). Skeletal muscle atrophy and increase intramuscular fat after incomplete spinal cord injury. Spinal Cord, 45, 304-309. doi:10.1038/sj.sc.3101968

Kirshblum, S. C., Burns, S. P., Biering-Sorensen, F., Donovan, W., Graves, D. E., Jha, A., ... Waring, W. 
(2011). International standards for neurological classification of spinal cord injury (Revised 2011). Journal of Spinal Cord Medicine, 34(6), 535-546. doi: 10.1179/204577211X13207446293695

Kottink, A. I. R., Oostendrop, L. J. M., Buurke, J. H., Nene, A. V., Hermens, H. J., \& Ijzerman, M. J. (2004). The orthotic effect of functional electrical stimulation on the improvement of walking in stroke patients with a dropped foot: A systematic review. Artificial Organs, 28(6), 577-586. PMID: 15153151

Li, F., Wu, Y., \& Li, X. (2014). Test-retest reliability and inter-rater reliability of the Modified Tardieu Scale and Modified Ashworth Scale in hemiplegic patients with stroke. European Journal of Physical and Rehabilitation Medicine, 50, 9-15.

Meier, R. H., Ruthsatz, D.C., \& Cipriani, D. (2014). Impact of AFO design on calf circumference. Lower Extremity Reviews, 6(10), 29-35.

van der Salm, A., Nene, A. V., Maxwell, D. J., Veltink, P. H., Hermens, H. J., \& Ijzerman, M. J. (2005). Gait impairments in a group of patients with incomplete spinal cord injury and their relevance regarding therapeutic approaches using functional electrical stimulation. Artificial Organs, 29(1), 8-14. PMID: 15644078

Singh, A., Tetreault, L., Kalsi-Ryan, S., Nouri, A., \& Fehlings, M. G. (2014). Global prevalence and incidence of traumatic spinal cord injury. Journal of Clinical Epidemiology, 6, 309-331.

Slijper, A., Danielsson, A., \& Willen, C. (2012). Ambulatory function and perception of confidence in persons with stroke with a custom-made hinged versus a standard ankle foot orthosis. Rehabilitation Research and Practice, 2012, 206495. doi: 10.1155/2012/206495. Vaught, S.L. (2001). Gait, balance and fall prevention. The Ochsner Journal, 3(2), 94-97.

Zou, D., He, T., Dailey, M., Smith K. E., Silva, M. J., Sinacore, D. R., ... Hastings, M. K. (2014). Experimental and computational analysis of composite ankle-foot orthosis. Journal of Rehabilitation Research and Development, 51(10), 1525-1536. doi: 10. 1682/ JRRD.2014-02-0046.

Zwaan, E., Becher, J. G., \& Harlaar, J. (2012). Synergy of EMG patterns in gait as an objective measure of muscle selectivity in children with spastic cerebral palsy. Gait \& Posture, 35, 111-115. doi: 10.1016/j.gaitpost.2011.08.019 